\title{
Comparative transcript profiling of resistant and susceptible peanut post-harvest seeds in response to aflatoxin production by Aspergillus flavus
}

Houmiao Wang ${ }^{1,2}$, Yong Lei ${ }^{1,2}$, Liyun Wan ${ }^{1,2}$, Liying Yan ${ }^{1,2}$, Jianwei Lv ${ }^{1,2}$, Xiaofeng Dai ${ }^{3}$, Xiaoping Ren ${ }^{1,2}$, Wei Guo ${ }^{3}$, Huifang Jiang ${ }^{1,2}$ and Boshou Liao ${ }^{1,2^{*}}$

\begin{abstract}
Background: Aflatoxin contamination caused by Aspergillus flavus in peanut (Arachis hypogaea) including in pre- and post-harvest stages seriously affects industry development and human health. Even though resistance to aflatoxin production in post-harvest peanut has been identified, its molecular mechanism has been poorly understood. To understand the mechanism of peanut response to aflatoxin production by A. flavus, RNA-seq was used for global transcriptome profiling of post-harvest seed of resistant (Zhonghua 6) and susceptible (Zhonghua 12) peanut genotypes under the fungus infection and aflatoxin production stress.
\end{abstract}

Result: A total of $128.72 \mathrm{~Gb}$ of high-quality bases were generated and assembled into 128, 725 unigenes (average length 765 bp). About 62, 352 unigenes (48.43\%) were annotated in the NCBI non-redundant protein sequences, NCBI non-redundant nucleotide sequences, Swiss-Prot, KEGG Ortholog, Protein family, Gene Ontology, or eukaryotic Ortholog Groups database and more than $93 \%$ of the unigenes were expressed in the samples. Among obtained 30, 143 differentially expressed unigenes (DEGs), 842 potential defense-related genes, including nucleotide binding site-leucine-rich repeat proteins, polygalacturonase inhibitor proteins, leucine-rich repeat receptor-like kinases, mitogen-activated protein kinase, transcription factors, ADP-ribosylation factors, pathogenesis-related proteins and crucial factors of other defense-related pathways, might contribute to peanut response to aflatoxin production. Notably, DEGs involved in phenylpropanoid-derived compounds biosynthetic pathway were induced to higher levels in the resistant genotype than in the susceptible one. Flavonoid, stilbenoid and phenylpropanoid biosynthesis pathways were enriched only in the resistant genotype.

Conclusions: This study provided the first comprehensive analysis of transcriptome of post-harvest peanut seeds in response to aflatoxin production, and would contribute to better understanding of molecular interaction between peanut and A. flavus. The data generated in this study would be a valuable resource for genetic and genomic studies on crops resistance to aflatoxin contamination.

Keywords: Arachis hypogaea, Post-harvest resistance, Aflatoxin production, Transcriptome

\footnotetext{
* Correspondence: liaoboshou@163.com

${ }^{1}$ Key Laboratory of Oil Crop Biology of the Ministry of Agriculture, Oil Crops

Research Institute of Chinese Academy of Agricultural Sciences, Wuhan 430062, China

${ }^{2}$ Chinese Academy of Agricultural Sciences-International Crop Research Institute for the Semi-Arid Tropics Joint Laboratory for Groundnut Aflatoxin Management, Oil Crops Research Institute of Chinese Academy of Agricultural Sciences, Wuhan 430062, China

Full list of author information is available at the end of the article
} 


\section{Background}

Peanut (Arachis hypogaea L.) is an important cash and oilseed crop and a key source of vegetable oil and protein worldwide. However, aflatoxin contamination caused by Aspergillus flavus and/or A. parasiticus has been a serious constraint to peanut industry, which is of great concern because aflatoxins are toxic, carcinogenic and teratogenic compounds associated with both acute and chronic toxicity in animal and human $[1,2]$. Infection of peanut by $A$. flavus occurs in both pre-harvest $[3,4]$ and post-harvest stages $[5,6]$. With appropriate drying, storing, processing, transporting and monitoring, healthy peanuts harvested from normal growth conditions are processed into secure and nutritious products for human/ animal consumption. Unfortunately, farmers in many developing countries in Asia and Africa, can't afford the cost associated with prevention, monitoring and mitigation of aflatoxin in peanut food/feed. Post-harvest aflatoxin contamination has led to an increased risk of exposure to aflatoxin resulting in outbreaks of acute aflatoxin poisoning [7] and increased morbidity in children suffering from stunted growth and malnutrition [8-10]. In addition, post-harvest aflatoxin contamination incurs significant economic costs, such as produce and market value losses, health care and associated disease surveillance, and for monitoring and mitigation of aflatoxin in peanut commodities [2, 11]. Thus, post-harvest aflatoxin contamination is an intractable problem in peanut products. Several management practices, including proper storage and transportation conditions, strict monitoring measures, and breeding cultivars for resistance to biotic and abiotic stresses, could prevent and/or reduce post-harvest aflatoxin contamination. Improvement of resistance to A. flavus invasion and/or aflatoxin production in peanut is considered to be the most cost-effective management approach. However, the resistance to post-harvest aflatoxin contamination in peanut hasn't been well understood.

The mycelia of $A$. flavus have to penetrate the peanut shell and seed coat before they reach the nutrient-rich cotyledons to derive sustenance. Resistance to aflatoxin contamination in peanut could be broadly classified into pod infection (shell), seed invasion (seed coat) and aflatoxin production (cotyledon) [12]. The first interaction between $A$. flavus and peanut is at the pod shell, which is a physical barrier, and the resistance is attributed to the shell structure. For post-harvest peanut, the resistance to pod infection is limited practical value, because ease of shelling is an important consideration in peanut industry. Moreover, the resistance of the pod shell to $A$. flavus infection would disappear when the shell is damaged or the peanut is shelled. The second barrier to this fungus is the seed coat, whose thickness, density of palisade layers, wax layers, and absence of fissures and cavities, are major contributors to the resistance to seed invasion. However, the seed coat would fail to resist $A$. flavus invasion when the testa is damaged or decorticated. A. flavus ultimately colonizes the cotyledons in the seed and produces the aflatoxin. Resistance to aflatoxin production is a very complex defensive mechanism affected by various biotic and abiotic factors. However, this kind of resistance to aflatoxin production, including the stress-responsive mechanism, is persistent and active $[13,14]$. To develop effective measures to combat postharvest aflatoxin contamination, it is important to investigate the molecular mechanisms of peanut resistance to aflatoxin production.

RNA-sequencing (RNA-seq) is a powerful and costefficient high-throughput technology for transcriptomic profiling that has been used successfully to interrogate the transcriptome of peanut in different development stages and response to various stresses [15-20]. With its higher sensitivity, RNA-seq could efficiently detect a larger range of dynamically expressed genes than microarrays. Furthermore, RNA-seq has been used to survey sequence variations and complex transcriptomes with low false-positive rates, and reproducibility [21]. Application of this technology has greatly accelerated understanding of the complexity of gene expression, regulation and networks [21], and has shown immense potential in explaining the molecular mechanism of host-resistance against pathogen infection. Peanut's resistance to Aspergillus colonization/aflatoxin production has been extensively reported, indicating that peanut has evolved a series of defense mechanisms against the fungi [22]. However, molecular mechanism of peanut resistance to aflatoxin production by $A$. flavus has been obscure.

To gain a comprehensive understanding of the molecular mechanism of resistance to aflatoxin production in post-harvest peanut seed, we used RNA-seq to obtain and compare transcriptomic profiles of a resistant genotype Zhonghua 6 and a susceptible genotype Zhonghua 12 in post-harvest seeds, with and without $A$. flavus inoculation, at the whole-genome level. De novo transcriptome assembly, functional annotation, and analysis of specific transcripts related to peanut's response to aflatoxin production by $A$. flavus were implemented. Differentially expressed genes and metabolic pathways associated with resistance to aflatoxin production were revealed by comparing $A$. flavus-inoculated and noninoculated seeds of the resistant/susceptible peanut genotypes. A better understanding of the molecular mechanism of resistance to aflatoxin production would aid in improving strategies to develop new resistant peanut cultivars. In addition, the transcriptomic information would aid functional genomics studies and further the understanding of resistant mechanisms to aflatoxin contamination in crops. 


\section{Results}

\section{Comparison of aflatoxin production in post-harvest} peanut seeds with fungal colonization

The aflatoxin content was quantified to define the response of Zhonghua 6 (resistant, R) and Zhonghua 12 (susceptible, $\mathrm{S}$ ) to aflatoxin production by $A$. flavus. Aflatoxin was not tested neither in $\mathrm{R}$ nor $\mathrm{S}$ on the $1^{\text {st }}$ day after incubation, and was tested starting from the $2^{\text {nd }}$ day after incubation in both $R$ and $S$ genotypes. The aflatoxin content increased significantly both in $\mathrm{R}$ and $\mathrm{S}$ after the $2^{\text {nd }}$ day after incubation; however, the trend of aflatoxin accumulation varied in the $\mathrm{R}$ and $\mathrm{S}$ genotypes (Table 1 ). In the $R$, the aflatoxin content increased most quickly between the $3^{\text {rd }}$ and $4^{\text {th }}$ day after incubation and then the increase ratio slowed down and the content became stable after the $7^{\text {th }}$ day. In the S, the aflatoxin content increased rapidly from the $3^{\text {rd }}$ to the $7^{\text {th }}$ day after incubation and then also remained stable. The aflatoxin content in the $\mathrm{R}$ was far lower than that in the $\mathrm{S}$ from the $2^{\text {nd }}$ day. At the peak of aflatoxin accumulation, the content in the $\mathrm{S}$ was over 10 -folds of that in the $\mathrm{R}$. Meanwhile, aflatoxin was not detected in non-inoculated $\mathrm{R}$ and $\mathrm{S}$ samples at all the 10 time points (Table 1). From the above experiment, the $\mathrm{R}$ possessed a desirable resistance to aflatoxin production in post-harvest seeds, while the $\mathrm{S}$ was highly susceptible.

\section{Transcriptome sequencing and de novo assembly}

The above aflatoxin content results suggested that peanut might alter their gene expression in response to aflatoxin production by $A$. flavus during incubation. The $1^{\text {st }}$, $3^{\text {rd }}$ and $7^{\text {th }}$ day after incubation were chosen as the inflection time points to study the defensive molecular metabolism of post-harvest seeds in response to aflatoxin

Table 1 The dynamic changes of aflatoxin content in the resistant genotype Zhonghua 6 and susceptible Zhonghua 12 during A. flavus colonization

\begin{tabular}{|c|c|c|c|c|}
\hline \multirow[t]{2}{*}{$\begin{array}{l}\text { Cultural } \\
\text { time (d) }\end{array}$} & \multicolumn{2}{|c|}{$\begin{array}{l}\text { Aflatoxin content in } \\
\text { Zhonghua } 6(\mu \mathrm{g} / \mathrm{kg})\end{array}$} & \multicolumn{2}{|c|}{$\begin{array}{l}\text { Aflatoxin content in } \\
\text { Zhonghua12 }(\mathrm{\mu g} / \mathrm{kg})\end{array}$} \\
\hline & CK & $\mathrm{T}$ & CK & $T$ \\
\hline 1 & 0 & $0 \pm 0$ & 0 & $0 \pm 0$ \\
\hline 2 & 0 & $1130.2 \pm 104.6$ & 0 & $4462.8 \pm 236.9$ \\
\hline 3 & 0 & $3175.5 \pm 232.8$ & 0 & $12687.1 \pm 720.2$ \\
\hline 4 & 0 & $12609.8 \pm 1226.4$ & 0 & $76671.9 \pm 6401.5$ \\
\hline 5 & 0 & $16906.0 \pm 1311.6$ & 0 & $111040.6 \pm 10125.6$ \\
\hline 6 & 0 & $19156.9 \pm 1608.0$ & 0 & $140227.3 \pm 11256.9$ \\
\hline 7 & 0 & $21107.6 \pm 1487.4$ & 0 & $195223.8 \pm 14354.4$ \\
\hline 8 & 0 & $21012.0 \pm 1441.2$ & 0 & $202425.0 \pm 14709.6$ \\
\hline 9 & 0 & $21059.8 \pm 1197.6$ & 0 & $193510.8 \pm 14805.0$ \\
\hline 10 & 0 & $20180.4 \pm 1501.8$ & 0 & $202632.5 \pm 14385.6$ \\
\hline
\end{tabular}

$T$ the peanut seed with inoculated $A$. flavus, $C K$ the peanut seed without inoculated $A$. flavus production. Therefore, 12 samples were used for transcriptome sequencing using Illumina HiSeq2000 system, comprising $\mathrm{R}$ and $\mathrm{S}$ genotypes with and without inoculation of $A$. flavus and sampled at $1 \mathrm{~d}, 3 \mathrm{~d}$ and $7 \mathrm{~d}$. We performed transcriptomic analysis of the 12 samples i.e., R_CK1, R_CK2, R_CK3, R_T1, R_T2, R_T3, S_CK1, S_CK2, S_CK3, S_T1, S_T2 and S_T3 (where CK is the non-inoculated control, and $\mathrm{T}$ indicates inoculated) with two biological replicates, to profile the peanut response to aflatoxin production (Table 2, Additional file 1). We obtained approximately 638.53 million raw reads for the $R$ samples (R_CK1, R_CK2, R_CK3, R_T1, R_T2, and R_T3) and 675.53 million raw reads for the $\mathrm{S}$ samples (S_CK1, S_CK 2, S_CK 3, S_T1, S_T2, and S_T3). After filtration of low-quality and adapter sequences, $128.72 \mathrm{~Gb}$ of clean bases remained in the 24 transcriptome libraries (Table 2, Additional file 1).

All the high quality reads were then used for de novo assembly of transcriptome data using the Trinity software. Using overlapping information in the high-quality reads, 406, 753 transcripts were generated, with an average length of 1, 577 bp and an N50 of 2, 629 bp (Table 3, Fig. 1, and Additional file 2-A). Under the clustering criteria of a minimum of 50 bp overlap and $90 \%$ identity, 128, 725 unigenes were obtained as a comprehensive reference data set of $A$. hypogaea (Table 3 ); further analysis was based on this final unigene data set. The length of unigenes ranged from 201 to $18,631 \mathrm{bp}$, with an average length of $765 \mathrm{bp}$; unigenes with lengths greater than 500 bp accounted for $39.36 \%$ of all unigenes (Table 3, Fig. 1, and Additional file 2-B).

\section{Gene annotation and functional classification of resistant and susceptible peanut transcriptome}

For validation and annotation of the assembled unigenes, all assembled unigenes were first screened against the NCBI non-redundant protein sequences $(\mathrm{Nr}), \mathrm{NCBI}$ non-redundant nucleotide sequences $(\mathrm{Nt})$, and SwissProt database using the NCBI blast 2.2.28+ program. Among the 128, 725 unigenes, 52, 691 (40.93 \%) had significant similarity to 39,488 unique proteins by $\mathrm{Nr}$ analysis. Of all the unigenes, 32, 396 (25.16 \%) with significant identities to Swiss-Prot proteins were matched with 17, 871 unique proteins accessions. In addition, 41, 555 (32.28 \%) unigenes had matches in the Nt database (Table 4). In total, 62, 352 unigenes (48.43\%) were annotated successfully in at least one of the Nr, Nt, SwissProt, KEGG Ortholog database (KO), Protein family (Pfam), Gene Ontology (GO), and eukaryotic Ortholog Groups (KOG) databases; 7, 061 unigenes (5.48 \%) were annotated in all seven databases. However, 66, 373 (51.56\%) unigenes had no matches in those databases. These un-matched unigenes may be novel genes or belong to untranslated regions, and might play specific 
Table 2 Summary of the sequence data from Illumina sequencing

\begin{tabular}{|c|c|c|c|c|c|c|c|}
\hline Library & Raw reads & Clean reads & Clean bases (Gb) & Error (\%) & Q20 (\%) & Q30 (\%) & GC content (\%) \\
\hline R_CK1_1 & 57130486 & 54867978 & 5.49 & 0.03 & 97.20 & 91.78 & 44.37 \\
\hline R_CK1_2 & 54713736 & 52859150 & 5.29 & 0.03 & 97.40 & 92.08 & 45.25 \\
\hline R_T1_1 & 55671406 & 55671406 & 5.57 & 0.03 & 97.26 & 91.79 & 45.19 \\
\hline R_T1_2 & 52776632 & 52776632 & 5.28 & 0.03 & 97.32 & 91.95 & 44.87 \\
\hline R_CK2_1 & 70575134 & 68662776 & 6.87 & 0.03 & 97.59 & 92.59 & 44.90 \\
\hline R_CK2_2 & 61656004 & 59697968 & 5.97 & 0.03 & 97.59 & 92.61 & 44.86 \\
\hline R_T2_1 & 62462134 & 62462134 & 6.25 & 0.03 & 97.66 & 92.77 & 44.40 \\
\hline R_T2_2 & 53206474 & 53206474 & 5.32 & 0.04 & 96.52 & 90.20 & 44.78 \\
\hline R_CK3_1 & 61917966 & 59434146 & 5.94 & 0.04 & 96.31 & 89.28 & 45.25 \\
\hline R_CK3_2 & 66649516 & 63956168 & 6.40 & 0.04 & 96.21 & 88.97 & 45.44 \\
\hline R_T3_1 & 23224306 & 23224306 & 2.32 & 0.05 & 94.80 & 87.41 & 46.27 \\
\hline R_T3_2 & 18549458 & 18549458 & 1.85 & 0.05 & 94.71 & 86.89 & 46.11 \\
\hline R-Total & 638533252 & 625368596 & 62.53 & & & & \\
\hline S_CK1_1 & 58045728 & 56103334 & 5.61 & 0.03 & 97.24 & 91.72 & 45.03 \\
\hline S_CK1_2 & 63689306 & 61798978 & 6.18 & 0.03 & 97.32 & 91.96 & 44.61 \\
\hline S_T1_1 & 57998512 & 57998512 & 5.80 & 0.03 & 97.27 & 91.85 & 44.24 \\
\hline S_T1_2 & 59669162 & 59669162 & 5.97 & 0.03 & 97.29 & 91.89 & 44.35 \\
\hline S_CK2_1 & 74137916 & 72280900 & 7.23 & 0.03 & 97.62 & 92.66 & 44.80 \\
\hline S_CK2_2 & 56939790 & 54259514 & 5.43 & 0.04 & 96.22 & 89.11 & 45.46 \\
\hline S_T2_1 & 49120232 & 49120232 & 4.91 & 0.04 & 96.27 & 89.28 & 44.59 \\
\hline S_T2_2 & 47405260 & 47405260 & 4.74 & 0.04 & 96.24 & 89.28 & 44.20 \\
\hline S_CK3_1 & 54341696 & 51982378 & 5.20 & 0.04 & 96.35 & 89.38 & 46.04 \\
\hline S_CK3_2 & 62599834 & 59709106 & 5.97 & 0.04 & 96.40 & 89.89 & 45.88 \\
\hline S_T3_1 & 48638388 & 48638388 & 4.86 & 0.04 & 96.21 & 89.17 & 44.96 \\
\hline S_T3_2 & 42948026 & 42948026 & 4.29 & 0.03 & 97.23 & 91.38 & 44.78 \\
\hline S-Total & 675533850 & 661913790 & 66.19 & & & & \\
\hline
\end{tabular}

R_CK1, R_CK2, and R_CK3: Zhonghua 6 without inoculated A. flavus cultured for 1 day, 3 days, and 7 days, respectively

R T1, R T2, and R T3: Zhonghua 6 with inoculated A. flavus cultured for 1 day, 3 days, and 7 days, respectively

S_CK1, S_CK2, and S_CK3: Zhonghua 12 without inoculated A. flavus cultured for 1 day, 3 days, and 7 days, respectively

S_T1, S_T2, and S_T3: Zhonghua 12 with inoculated A. flavus cultured for 1 day, 3 days, and 7 days, respectively

Q20: The percentage of bases with a Phred value $>20$

Q30: The percentage of bases with a Phred value $>30$

roles in stress response to aflatoxin production by $A$. flavus in peanut seeds.

To identify the functional categories of the annotated unigenes, GO, KOG, and KEGG were used to classify the unigenes annotated by known proteins. In total, 40, 889 unigenes with Blast2GO matches to known proteins were assigned to a broad range of GO terms (Table 4, Fig. 2a, and Additional file 3). The majority of the unigenes were assigned to "Molecular function" (27, 630;
$67.57 \%)$, followed by "Biological process" (27, 092; $66.26 \%)$ and "Cellular component" (17, 434; 42.64 \%). A total of 17, 798 unigenes were annotated using the KOG database (Table 4), and these unigenes were assigned to 26 KOG categories (Fig. 2b, and Additional file 3). Among the 26 KOG categories, the cluster related to "General function prediction only" (3, 218; $18.08 \%)$ was the largest group, followed by "Posttranslational modification, protein turnover, chaperones" (2, 068; $11.62 \%)$

Table 3 Summary of the de novo assembly results using Trinity

\begin{tabular}{|c|c|c|c|c|c|c|c|c|c|}
\hline \multirow[t]{2}{*}{ Category } & \multicolumn{4}{|l|}{ Number } & \multirow{2}{*}{$\begin{array}{l}\text { Total } \\
\text { number }\end{array}$} & \multirow{2}{*}{$\begin{array}{l}\text { Mean } \\
\text { length (bp) }\end{array}$} & \multirow{2}{*}{$\begin{array}{l}\text { N50 } \\
\text { value }\end{array}$} & \multirow{2}{*}{$\begin{array}{l}\text { N90 } \\
\text { value }\end{array}$} & \multirow{2}{*}{$\begin{array}{l}\text { Total } \\
\text { nucleotides }\end{array}$} \\
\hline & $200-500 \mathrm{bp}$ & 500-1000 bp & $1000-2000 \mathrm{bp}$ & $2000 \mathrm{bp}$ & & & & & \\
\hline Transcripts & 117,970 & 74,290 & 94,186 & 120,307 & 406,753 & 1,577 & 2,629 & 755 & $641,557,533$ \\
\hline Unigenes & 78,055 & 25,178 & 14,146 & 11,346 & 128,725 & 765 & 1,355 & 293 & $98,499,770$ \\
\hline
\end{tabular}




\section{Length Distribution}

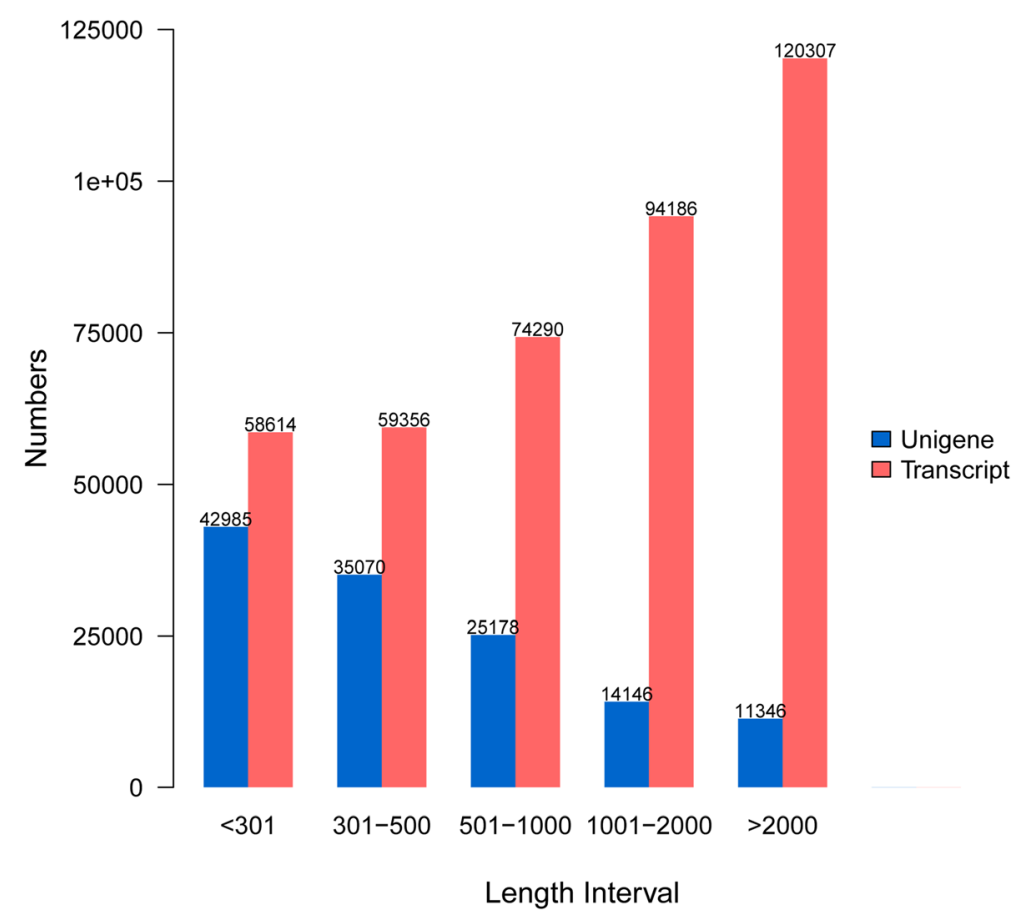

Fig. 1 Length distribution of unigenes (blue) and transcripts (red)

and "Signal transduction mechanisms" (1, 415; $7.95 \%)$. Additionally, all the unigenes were analyzed with the KEGG pathway database, 13, 196 (10.25 \%) with significant matches in the database and were assigned to five main categories, which included 32 sub-categories and 273 KEGG pathways (Table 4, Fig. 2c, and Additional file 3). Among the 32 sub-categories, "Carbohydrate metabolism" was the sub-category with the greatest number of unigenes (1, 550; $11.75 \%)$, followed by "Translation" (1, 218; $9.23 \%)$ and "Amino acid metabolism" (1, 115; $8.45 \%)$. These

Table 4 Statistics of the functional annotation of assembled unigenes

\begin{tabular}{lll}
\hline Public database & Number of unigenes & Percentage (\%) \\
\hline $\mathrm{Nr}$ & 52,691 & 40.93 \\
$\mathrm{Nt}$ & 41,555 & 32.28 \\
Swiss-Prot & 32,396 & 25.16 \\
$\mathrm{GO}$ & 40,889 & 31.76 \\
$\mathrm{KOG}$ & 17,798 & 13.82 \\
Pfam & 35,318 & 27.43 \\
KO & 13,196 & 10.25 \\
All Databases & 7,061 & 5.48 \\
Annotated in at least & 62,352 & 48.43 \\
one Database & & 100 \\
Total Unigenes & 128,725 & \\
\hline
\end{tabular}

annotations and classifications provided a valuable resource for investigating specific processes, functions and pathways of the identified unigenes.

Identification and analysis of differentially expressed genes Fragments Per Kilobase of transcript sequence per Millions base pairs sequenced (FPKM) was used to quantify the transcript levels of the reads, which facilitated the comparison of mRNA levels both within and between samples [23]. The assembled set of 128, 725 unigenes was used as the reference onto which clean reads from each library were mapped to generate a putative expression profile for the transcripts (Additional file 4). All the 128, 725 unigenes were normalized and calculated by the FPKM method using uniquely mapped reads (Additional files 5 and 6). Unigenes with FPKM value $>0.3$ were considered to be transcriptionally expressed [24]. Among the unigenes, $93.16 \%(119,917)$ were expressed in at least one of samples and 19, 230 unigenes were expressed in all 24 libraries (Additional file 5). The expressed unigenes data were highly reproducible between two biological replicates in both $\mathrm{R}$ and $\mathrm{S}$ genotypes, although a certain number of specifically expressed unigenes were obtained from each biological replicate (Additional files 5 and 7). To validate the RNA-Seq digital expression data, 20 expressed unigenes were 


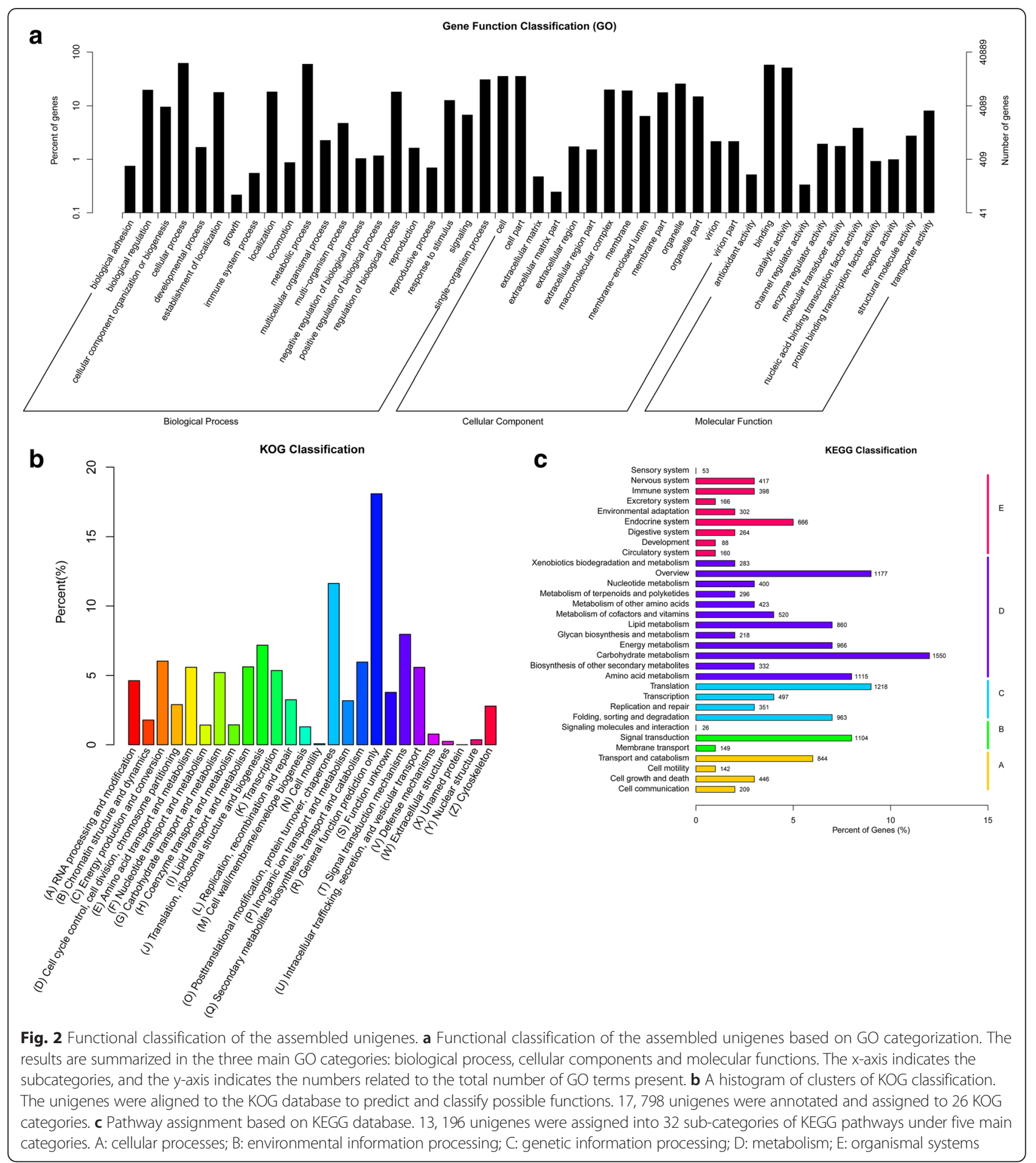

randomly selected, primers were designed (Additional file 8) and quantitative real-time reversed transcription PCR (qRT-PCR) was performed. The results showed a high correlation $\left(R^{2}=0.714\right.$; Additional file 9) between the RNA-seq and qRT-PCR data, which confirmed the authenticity of these expressed unigenes and the transcriptome analysis.
Further, DESeq was used to identify the differentially expressed genes (DEGs) across the samples where only those unigenes with the corrected $p(q)$ value $<0.05$ were considered differentially expressed [25]. The differential comparisons between the control and the inoculated samples identified DEGs that responded to aflatoxin production in both genotypes; the comparison between 
the inoculated samples identified DEG $\mathrm{s}$ between the $\mathrm{R}$ and $\mathrm{S}$ genotypes in response to aflatoxin production (Additional file 10). An important proportion of DEGs $(30,143)$ were identified in the comparisons among the three time points in both genotypes (Additional files 11 and 12). We observed that the up-regulated and downregulated DEGs showed similar change trends through the three time points (Additional files 10 and 11). The number of up-regulated DEGs was markedly higher than the down-regulated in comparisons of the control and inoculated samples of both genotypes. There were more up-regulated DEGs in the R genotype than in the $\mathrm{S}$ at each time point, while there were fewer down-regulated DEGs in the $\mathrm{R}$ genotype than in the $\mathrm{S}$ at each time point. To obtain a global view of the gene expression patterns, we performed hierarchical clustering of all the DEGs based on the $\log _{10}$ FPKMs for the 12 samples (Additional files 13 and 14). The results showed that the DEGs data were highly reproducible between two biological replicates in both R and S genotypes (Additional file 14). Similar expression patterns were found in the earlier inoculated samples (R_T1 and S_T1); and distinct sample-specific expression patterns were observed in each genotype at the latter two time points (Additional file 13).

\section{Functional classification of differentially expressed genes}

To analyze the functions of the DEGs, a GO analysis was performed using GOseq method in Blast2GO [26]. GO terms with corrected $p(q)$ value $<0.05$ were considered significantly enriched among the DEGs. GO enrichment analysis of the up-regulation DEGs in the inoculated sample were compared with the control at paired time points of $\mathrm{R}$ and $\mathrm{S}$ genotypes, respectively. Many significantly enriched terms in the biological process, molecular function, and cellular component categories were identified (Additional file 15). Metabolic progress (GO:0008152), catalytic activity (GO:0003824), and oxidation-reduction progress (GO:0055114) were dominant terms in comparisons of the $A$. flavus-inoculated (treatment) to noninoculated (control) (R_T1vs. R_CK1, R_T2 vs. R_CK2, R_T3 vs. R_CK3, S_T2 vs. S_CK2, and S_T3 vs. S_CK3). Many other common/unique terms affected by aflatoxin production were enriched in the treatments versus the controls of the R and S genotypes, whereas no GO terms were enriched in the comparison of S_T1 vs. S_CK1. Notably, the terms antioxidant activity (GO:0016209), phenylpropanoid biosynthetic process (GO:0009699), peroxidase activity (GO:0004601), linoleate 13S-lipoxygenase activity (GO:0016165), oxylipin metabolic progress (GO:0031407), coumarin biosynthetic process (GO:0009805), stilbene biosynthetic process (GO:0009811), cinnamic acid biosynthetic process (GO:0009800) and flavonoid biosynthetic process (GO:0009813), which have key roles in plant resistance to pathogens [27], were exclusively present in the $\mathrm{R}$ genotype at the $3^{\text {rd }}$ day after incubation $\left(\mathrm{R}_{-} \mathrm{T} 2\right.$ vs. R_CK2). This indicated that metabolisms involving a series of lipids and secondary metabolites were quite active in the complex resistance processes of the R genotype in response to aflatoxin production. Concurrently, a GO analysis was conducted for the down-regulated DEGs in the inoculated samples of the $\mathrm{R}$ and $\mathrm{S}$ genotypes, respectively (Additional file 15). However, the analysis failed to confirm enrichment in any term in the down-regulated DEGs data obtained from R_T1 vs. R_CK1, S_T1 vs. S_CK1, R_T2 vs. R_CK2, and S_T2 vs. S_CK2. The results suggested that the metabolisms of peanut were activated by $A$. flavus colonization at an early stage of the peanut $-A$. flavus interaction process. When comparing T3 with CK3, protein binding (GO:0005515) and protein folding (GO:0006457) were the dominant GO terms of the downregulated DEGs in comparisons of both genotypes at the $7^{\text {th }}$ day after incubation. The induction of defense is costintensive and contact with pathogens would greatly alter host-plant metabolism [28, 29]. Great changes were observed in gene expression of both $\mathrm{R}$ and $\mathrm{S}$ genotypes during the peanut $-A$. flavus interaction. Nonetheless, the GO analysis showed more active response in the $\mathrm{R}$ genotype than in the $\mathrm{S}$.

To further investigate the biological functions and interactions of genes, pathway-based analysis was conducted using KEGG [30]. All DEGs obtained from the comparisons of the treatment versus the control in $\mathrm{R}$ and $\mathrm{S}$ genotype at three paired time points were analyzed using KOBAS 2.0 to identify their associated KEGG metabolic pathways [31]. Eighteen pathways were significantly up-regulated in comparisons of the treatment versus the control in both genotypes, while 37 pathways were significantly repressed by aflatoxin production ( $q$ value $<0.05$ ) (Additional file 16). However, KEGG metabolic pathway analysis failed to confirm enrichment in any up-regulated pathway obtained in R_T3 vs. R_CK3, and S_T3 vs. S_CK3, which indicated that many metabolic pathways in post-harvest peanut seeds might be repressed by a mass of A. flavus mycelia and/ or aflatoxin. Not unexpectedly, several up-regulated pathways, such as "phenylpropanoid biosynthesis", "flavonoid biosynthesis", and "stilbenoid, diarylheptanoid and gingerol biosynthesis", were uniquely enriched in the R genotype at the $3^{\text {rd }}$ day after inoculation. This analysis is consistent with the previous observation that the fungus attack can influence a broad range of pathways and a large proportion of the genes in the transcriptional networks are affected [20, 32-35].

\section{Expression analysis of defense-related genes in peanut response to aflatoxin production by $A$. flavus}

Analyzing the expression profiles of $\mathrm{R}$ and $\mathrm{S}$ genotypes in response to aflatoxin production by A. flavus, especially 
the 30, 143 unigenes that were significantly differentially transcribed (Additional file 11), we detected 842 potential defense-related genes involved in peanut response to aflatoxin production (Additional file 17). These defenserelated genes encoded nucleotide binding site-leucine-rich repeat proteins (NBS-LRR), leucine-rich repeat receptorlike kinase (LRR-RLK), mitogen-activated protein kinase (MAPK), transcription factors (TFs), pathogenesis-related (PR) proteins, and crucial factors of other defense related pathways (Additional file 17).

Ninety DEGs with NBS-LRR domains were identified in this study. The NBS-LRR genes were all downregulated at the first time point ( $1^{\text {st }}$ day after incubation) in $\mathrm{R}$ genotype, whereas there were five up-regulated $N B S-L R R$ genes in $\mathrm{S}$ genotype at the first time point; at the second time point ( $3^{\text {rd }}$ day after incubation), all $N B S-L R R$ genes were up-regulated in both genotypes; about a half of NBS-LRR genes in $\mathrm{R}(54.84 \%)$ and $\mathrm{S}$ $(55.56 \%)$ were up-regulated at the third time point $\left(7^{\text {th }}\right.$ day after incubation). Overall, the expression levels of $N B S-L R R$ genes in $\mathrm{R}$ genotype were higher or slightly higher than in $\mathrm{S}$. Furthermore, the expression patterns of 19 DEGs encoding chitinases and 84 DEGs involving in the lectins metabolic pathway were similar to the differentially expressed NBS-LRRs. Though the differentially expressed $L R R-R L K s$ (143) showed up- or downregulation in each comparison, there were up-regulated $L R R-R L K \mathrm{~s}$ than down-regulated ones in both genotypes. Interestingly, 28 DEGs involved in MAPK cascades including 6 ones were identified at the first two time points and another 22 ones were obtained at the third time point. Two up-regulated DEGs (comp90525_c1 and comp77989_c0) encoding extracellular signal-regulated kinase 1/2 (K04371) and one up-regulated DEG (comp 90797_c0) encoding MAPK kinase 1 (K04368) were identified in both genotypes at the third time point, whose expression level was higher in $\mathrm{R}$ genotype than in $\mathrm{S}$. Additionally, 71 ARFs (ADP-ribosylation factors), 19 LOXs (lipoxygenases) and 6 PGIPs (polygalacturonase inhibitor proteins) differentially expressed in the $\mathrm{T} v s$. $\mathrm{CK}$, and most of them were up-regulated after A. flavus inoculation in both genotypes. Compared with $\mathrm{S}$ genotype, $10 A R F \mathrm{~s}, 13$ LOXs and 6 PGIPs were induced to a higher level in the R.

Fifty-eight DEGs encoding WRKY transcription factors (TFs) were identified, and all of them were up-regulated in both genotype. The number of expressed WRKYs gradually increased with incubated time in both genotypes, 2 and $21 W R K Y$ S were respectively identified at the first and the third time point. The transcript levels of those WRKYs were higher in $\mathrm{R}$ genotype than in $\mathrm{S}$. As for bZIP TFs, 65 differentially expressed bZIPs were identified; however, no $b Z I P$ was found at the second time point in both genotypes. Two bZIPs were identified at the first time point, but there was no significant difference in the expression levels between $\mathrm{R}$ and $\mathrm{S}$ genotypes. Twenty-three bZIPs were found at the third time point, and the transcript levels in R genotype were much higher than in S. Additionally, 67 DEGs encoding ethylene-responsive TFs (ERFs) were identified. All ERFs were down-regulated in $\mathrm{R}$ or $\mathrm{S}$ genotype at the first time point; this down-regulation was more severe in $\mathrm{S}$ genotype than in R. Six $E R F$ s were up-regulated at the second time point, and 3 of them were identified in both genotypes, which expression levels in $\mathrm{S}$ genotype was higher than in R. At the third time point, 43 up- and 22 downregulated $E R F$ s were identified, and 29 of all ones were found in both genotypes.

A total of 86 DEGs were annotated as PR proteins in $\mathrm{R}$ and $\mathrm{S}$ genotypes, including PR-1, PR-2, PR-5, PR-10, PR-sth2, PR-Bet VI family and other resistance proteins. Thirteen DEGs encoding PR protein Bet VI family and 4 DEGs encoding PR protein STH2 showed greater expression changes in $\mathrm{R}$ genotype compared with the $\mathrm{S}$. One DEG (comp91631_c1) annotated as PR-10, was identified in $\mathrm{R}$ genotype at the latter two time points, while the DEG was identified in S genotype only at the third time point. Additionally, one DEG was annotated as PR-2 only in R genotype, and the PR-1 and PR-5 genes were only identified in $\mathrm{S}$. Most $P R$ genes were induced to a higher level in R genotype compared with the $\mathrm{S}$.

We identified 45 DEGs involved in phytohormonal metabolism and signaling pathways that were up- or downregulated in response to aflatoxin production, including salicylic acid (SA), ethylene (ET), and abscisic acid (ABA). Six DEGs were involved in the SA signaling pathway in both genotypes, and 2 of them (comp80400_c0 and comp91788_c0) encode pathogen-inducible salicylic acid glucosyltransferase (K13691). It is interesting to note that one DEG (comp78095_c0), encoding salicylic acid methyltransferase-like protein (NM_001250193.1), was identified only in R genotype. Moreover, 6 DEGs involving in the ET signaling pathway were identified. Although all ET-related DEGs were down-regulated, most of them were repressed more severely in $\mathrm{R}$ genotype than in $\mathrm{S}$. Similarly, 30 DEGs involving in the ABA signal pathway were identified. There were 2, 3 and 3 DEGs respectively encode the key enzymes aldehyde oxidase (AO), 9-cisepoxycarotenoid dioxygenase (NECD) and Zeaxanthin epoxidase (ZEP) of ABA biosynthesis. $A O, N E C D$ and $Z E P$ were up-regulated at the first time point, and down regulated at the third time point in $\mathrm{R}$ genotype. While in $\mathrm{S}$ genotype, the expression of $A O, N E C D$ and $Z E P$ were upregulated to various extents after $A$. flavus inoculation. Additionally, anther 17 ABA-related DEGs encoding ABA 8 '-hydroxylase (7), ABA-insensitive protein (3), ABA receptor (6) and ABA response element binding protein (1) were subjected to up- or down-regulation to various extents in $\mathrm{R}$ and $\mathrm{S}$ peanut seeds after $A$. flavus colonization. 
There were 38 DEGs involving in the biosynthesis of plant phenylpropanoid- derived compounds. Our analysis showed that the expression of DEGs encoding phenylalanine ammonia-lyase (PAL), cinnamate 4-hydroxylase $(\mathrm{C} 4 \mathrm{H})$ and 4-coumarateCoA ligase (4CL) were up-regulated in both genotypes after $A$. flavus inoculation. PAL, $\mathrm{C} 4 \mathrm{H}$ and 4CL catalyze the first three steps of phenylpropanoid- derived compounds biosynthesis, the general phenylpropanoid pathway (GPP) [36]. The products of GPP then serve as precursors for diverse phenylpropanoid-derived compounds. Five DEGs encoding PAL were identified in both genotypes that were induced to higher levels in $\mathrm{R}$ genotype than in S. Three PALs (comp75395_c0, comp 81599_c0, and comp83560_c0) were activated by aflatoxin production at all three time points in $\mathrm{R}$ genotype, and their changes in expression levels at the latter two time points were significantly higher than at the first time point. While in comparisons of S genotype, no one and 6 differentially expressed PALs were identified at the first two time points and at the third time point, respectively. Differentially expressed C4Hs (4) and 4CLs (9) were identified and showed the same expression patterns as PALs. We also investigated chalcone synthase (CHS), the entry point of the flavonoid pathway, and its close relative stilbene synthase (STS), the key enzyme of stilbenes biosynthesis. In total, 19 DEGs encoding CHS and 3 differentially expressed STSs were up-regulated in both genotypes. The expression patterns of CHSs and STSs were similar to the DEGs encoding the key enzymes of GPP. The results showed that DEGs involving in phenylpropanoid pathway were induced earlier and at a higher level in R genotype compared with the S.

\section{Discussion}

Peanut is an important economic and nutritional crop, and is one of the most susceptible crops to colonization by $A$. flavus and subsequent aflatoxin contamination. A better understanding of molecular mechanism for resistance to aflatoxin contamination will aid designing strategies to develop new peanut cultivars with improved resistance. Transcriptomic analysis is a crucial research approach, as it not only helps in large-scale identification of mRNAs, but also provides insights into the molecular basis of genes involved in plant physiological and pathological processes. In this study, RNA-seq was used to interrogate transcriptome of $A$. hypogaea to explore the molecular mechanism of resistant and susceptible genotypes response to aflatoxin production by A. flavus. A large number of $A$. hypogaea transcriptomic unigenes $(128,725)$ were obtained and about half of the unigenes $(62,352 ; 48.43 \%)$ were annotated successfully in at least in Nr, Nt, Swiss-Prot, GO, KOG, and KEGG databases. As far as we know, this is the first report to identify large numbers of genes involved in different metabolic pathways in post-harvest peanut seeds in response to aflatoxin production using RNA-seq technology. What's more, the total clean reads and unigenes, N50 value, and average length of the unigenes reported here were far greater than those previous transcriptomic profiling reports on the developing peanut seed [20,37,38]. A large percentage unigenes $(51.57 \%$ ) could not be annotated in the present study because of technical limitations (such as sequencing depth or read length) [39] and the absence of genomic information on A. hypogaea [15], which are common to all studies that perform de novo transcriptomic analysis. Transcriptome sequences are valuable resource, especially for species without a completely sequenced genome, such as the cultivated peanut. Our results enriched the genomic information on $A$. hypogaea in public databases, and laid a foundation for the evaluation and understanding of post-harvest peanut seed in response to aflatoxin production.

Previous studies on plant resistance to A. flavus infection/aflatoxin production mainly focused on actively developing seeds during the pre-harvest time course $[1,17,20,35,40]$, and only few reports examined postharvest peanut seeds $[41,42]$. Thus, our study on postharvest peanut seeds response to aflatoxin production could contribute to a comprehensive understanding of the mechanism of resistance to aflatoxin contamination. In the comparisons analyzed, including among the three time points of both genotypes, we identified 30, 143 DEGs. There were markedly more up-regulated DEGs than down-regulated ones in the comparisons between the treatments versus the controls in both genotypes. In addition, the number of up-regulated DEGs in R genotype was higher than in $\mathrm{S}$ at each time point. The results suggested that aflatoxin production activated/repressed the expressions of many genes in $\mathrm{R}$ and $\mathrm{S}$ genotypes. Genes involved in defensive reactions to aflatoxin production were activated to higher levels in $\mathrm{R}$ genotype compared with in S.

Peanut has evolved sophisticated defense mechanisms to combat pathogen invasion, such as blocking pathogen invasion and activating a range of defense responses [22, 43]. A. flavus is a facultative parasite that behaves as both a biotroph and a necrotroph [44]. The molecular mechanisms of plant defense against facultative parasites are relatively sophisticated [43]. The mechanism of peanut's resistance to aflatoxin production was quite complex, with many defense-related DEGs showing transcriptional differences between $\mathrm{R}$ and $\mathrm{S}$ genotypes in our study. Successful colonization of plant tissues by microbial pathogens requires overcoming the cell wall. To this end, pathogens produce a wide array of plant cell wall degrading enzymes [45]. Polygalacturonases (PGs) cleave the $\alpha-(1-4)$ linkages between the D-galacturonic acid residues of homogalacturonan, causing cell separation in the host tissue. To 
counteract the activity of PGs, plants deploy cell wall PGIPs that specifically inhibit the pectin-depolymerizing activity of PGs [46]. In addition to PGs inhibition, the interaction between PGs and PGIPs promotes the formation of oligogalacturonides, which are elicitors of a variety of defense responses [47]. Our analysis showed that all 6 differentially expressed PGIPs were induced to a much higher level in $\mathrm{R}$ genotype than in $\mathrm{S}$, indicating that the PGIPs probably play a more significant role in the defense response to aflatoxin production in $\mathrm{R}$ genotype. LRRRLKs, a large family of signaling proteins comprising extracellular repeats that are linked by a transmembrane domain to either an intracellular adapter domain or a kinase domain [48], take part in a variety of different pathological processes [49]. About 140 differentially expressed $L R R-R L K \mathrm{~s}$ were identified in peanut seeds responding to aflatoxin production by $A$. flavus, and many more $L R R$ $R L K$ s were up regulated in the defensive reactions. FLS2 is a typical pattern recognition receptor (PRR), which can activate the MAPK cascade [50]. Plant MAPK cascades are involved in signaling multiple defense responses, including the biosynthesis and signaling of plant stress and defense hormones, reactive oxygen species (ROS) generation, stomatal closure, defense gene activation, phytoalexin biosynthesis, cell wall strengthening, and the hypersensitive response (HR) cell death [50, 51]. Twenty-eight DEGs involved in MAPK cascades were induced at a higher level in the R genotype after A. flavus colonization compared with in $S$.

Several TFs and other key regulators of plant immunity are induced by the activation of the MAPK cascades $[15,52]$. The TFs of the WRKY, bZIP, and ERF families, which have been proven to be involved in plant defense responses [53], were analyzed in this study. The transcription of $W R K Y \mathrm{~S}$ is strongly and rapidly up-regulated in response to pathogen invasion and wounding in numerous plant species [54]. Almost all DEGs (58) encoding WRKY proteins were up-regulated, and the activated expression levels of those WRKYs were higher in $\mathrm{R}$ than in S. Similar to WRKY, the bZIP proteins form a super family of TFs that mediate plant stress responses [55]. Moreover, a bZIP transcription factor, AtfB, is a key player in the coordinated expression of antioxidant genes and genes involved in aflatoxin biosynthesis [56]. We 65 differentially expressed $b Z I P$ s, but none of them encoded AtfB; however, the transcript levels of 23 bZIPs were much higher in $\mathrm{R}$ genotype than in $\mathrm{S}$. The ERF family is found only in the plant kingdom, and includes several genes involved in regulation of disease resistance pathways [57]. ERFs probably participate in the regulation of aflatoxin production resistant pathways in peanut seeds similarly to WRKY and bZIP, because most ERFs were significantly up-regulated after A. flavus inoculation. Additionally, most $A R F \mathrm{~s}$ were up-regulated after $A$. flavus inoculation and 10 of them were up-regulated to higher levels in $\mathrm{R}$ compared with in S. The ARFs are family of monomeric GTP-binding proteins belonging to the small GTPases of the Ras super-family, which regulate a wide variety of physiological and pathological processes in various plants [58]. Like all small G-proteins, ARFs functions as molecular switches that alternate between a GTP and membrane-bound "on" state and a GDP-bound, mostly cytosolic "off" state [59]. ARFs might also switch the expression of some target genes involved in peanut's response to aflatoxin production.

The presence of ABA, SA, and ET phytohormonal pathways in peanut seeds could be concurrent with their response to aflatoxin production by mediating and channeling many stress-responsive genes that help plants to survive stress [60]. ABA is considered as a negative regulator of disease resistance [61, 62]. Consistent with previous reports, the DEGs involved in $\mathrm{ABA}$ production and signaling pathway were expressed at high levels in $\mathrm{S}$ genotype than in R. Almost all biotic and abiotic stress conditions elicit ET synthesis in plants [63], moreover, ET could inhibit aflatoxin biosynthesis in A. flavus on A\&M medium [64]. However, all DEGs involved in ET production and signal pathway were down-regulated in response to aflatoxin production, and most of them were repressed to a higher level in $\mathrm{R}$ than in S. Depending on the pathogen, disease symptoms seem to be either reduced or enhanced by ET, or not affected, in different plants [63]. We deduced that ET may suppress peanut's ability to resist aflatoxin production; however, this deduction needs further confirmation. SA plays a crucial role in plants and has a suppressive effect on some fungi [65]. Recent research showed that SA inhibits the mycelial growth and mycotoxin production of A. flavus in vitro and in vivo [66]. Six DEGs involved in the SA signaling pathway in both genotypes, and showed similar expression patterns, except for comp78095_c0, encoding salicylic acid methyltransferase-like protein (NM_001 250193.1), which was identified only in $R$ genotype. This DEG might be associated with the resistance of $R$ genotype. Phytohormones are involved in mediating fungusplant interactions, and their roles are totally different [61]. Consistent with previous reports, our transcriptomic analysis showed different expression patterns of genes involved in phytohormone production and signaling in response to aflatoxin production.

NBS-LRR genes are the most represented group of plant disease resistance genes which are key component in the interactions between plants and pathogens $[43,67]$. Two groups of NBS-LRR genes, CC-NBS-LRR and TIR-NBS$L R R$, were identified in both genotypes and showed a general up-regulation after $A$. flavus inoculation. We also found that the expression patterns of those DEGs involved in chitinases and lectins biosynthesis were similar to 
differentially expressed NBS-LRRs. Plant chitinases [68, 69] and lectins [70] are considered to be involved in the plant defense against $A$. flavus [40,71]. Our results indicated that NBS-LRR, chitinases and lectins probably play important roles in inhibiting aflatoxin production; these DEGs had different responsive reactions between in $\mathrm{R}$ and $\mathrm{S}$ genotypes. POD proteins, as well as other regulators of peanut immunity [72], contributed to the response to aflatoxin production. Differentially expressed $P O D$ s showed a significantly higher expression in $\mathrm{R}$ compared with in $\mathrm{S}$. The expression of POD, an oxidative radical scavenging enzyme, indicated a better management of oxidative radicals in $\mathrm{R}$ genotype during aflatoxin production process. Oxylipins play important roles in the aflatoxin biosynthesis [73]; and 13S-HPODE inhibits aflatoxin production by $A$. flavus [1]. We identified 19 DEGs encoding LOXs and most of them were up-regulated, moreover, "linoleate 13S-lipoxygenase activity", and "oxylipin biosynthetic process" were enriched in the R genotype. LOXs could affect aflatoxin production [73], and 13-LOXs and their oxidative products could be involved in the defense response to aflatoxin production in the post-harvest peanut seeds.

PR proteins have been defined as proteins encoded by the host plant but induced by various types of pathogens, such as fungi, bacteria, viruses, and also by the application of chemicals that mimic the effect of phytopathogen infection or induce similar stresses [61,74, 75]. The expressions of PR-1, PR- 4 and PR-10 were induced to a higher level to trigger the rapid activation of defense-responsive mechanisms in many fungus-challenged plant species, such as wheat, rice, maize, Arabidopsis [61, 76, 77]. Eighty-six DEGs were annotated as PR proteins in $\mathrm{R}$ and S genotypes, including PR-1, PR-2, PR-5, PR-10 and other resistance proteins. PR-1 family is induced by SA and pathogens, and is commonly used as a marker for systematic acquired resistance [78]. The PR-2 family consists of $\beta$-1, 3-glucanases and catalyzes the hydrolysis of $\beta$-1,3-glucans, which act in fungal defense by hydrolyzing fungal cell walls and by generating elicitors [75]. The PR-5 family includes permatins, zeamatins and thaumatin-like proteins, which cause osmotic breakage of transmembrane pores on fungal plasma membranes [75]. PR-10 proteins are small and structurally conserved, but have diverse role in stress signaling [75]. Furthermore, PR-10 proteins have positive roles in maize resistance to $A$. flavus growth and aflatoxin production [76].

Plant phenylpropanoid-derived compounds are a diverse family of phenylalanine-derived secondary metabolites, which include flavonoids, stilbenes, monolignols, and various phenolic acids [79]. Among their many functions in plants, phenylpropanoid compounds play important roles in resistance to pathogen attack [27], moreover, flavonoids and stilbenoids inhibit A. flavus development and aflatoxin production [41, 80-82] The key enzymes, PAL, C4H, 4CL, CHS and STS, of the phenylpropanoid biosynthetic pathway were up-regulated in $\mathrm{R}$ and $\mathrm{S}$ genotypes after $A$. flavus inoculation. The DEGs involving in phenylpropanoid biosynthesis pathway were induced to higher levels in $\mathrm{R}$ genotype earlier than inS. In addition, the terms "phenylpropanoid biosynthesis", "flavonoid biosynthesis", and "stilbenoid, diarylheptanoid and gingerol biosynthesis" were enriched only in the $R$ genotype. These data suggested that phenylpropanoidderived compounds biosynthesis might be closely associated with resistance to aflatoxin production in postharvest peanut seed. Having identified these candidate genes, further research will be required to determine whether these DEGs are responsible for the difference in resistance to aflatoxin production between $\mathrm{R}$ and $\mathrm{S}$ peanuts.

Although $\mathrm{R}$ and $\mathrm{S}$ genotypic peanuts both underwent a large transcriptional modulation representing the various metabolic processes involved in defense against aflatoxin production, many more resistance-related DEGs were significantly up-regulated and enriched in $R$ genotype, which suggested that $\mathrm{R}$ genotype possessed comprehensive and prompt responses toward the biotic stress. These transcriptional modulations could eventually result in the synthesis of resistance-related proteins, secondary metabolites and signaling molecules that provide defensive advantages to the peanut. Further investigations are necessary to characterize the biosynthesis of these molecules and their molecular mechanisms in response to A. flavus colonization and aflatoxin production in the peanut. The comprehensive analysis of their transcriptional profiles under aflatoxin production stress will strengthen the understanding of the genes and metabolic pathways involved in resistance to aflatoxin production, and will provide direction for the future studies on the molecular mechanisms of resistance to aflatoxin contamination in peanut.

\section{Conclusions}

In the present study, RNA-seq was applied to conduct a global characterization of the resistant and susceptible peanut transcriptomes in response to aflatoxin production by $A$. flavus. In total, $128.72 \mathrm{~Gb}$ clean bases were obtained and 128, 725 unigenes were assembled from 24 libraries of post-harvest peanut seeds. A number of DEGs were activated or repressed by aflatoxin production in the $R$ and $S$ genotypes, more DEGs were up-regulated in the R genotype than in the $\mathrm{S}$ at every time point. Furthermore, 842 putative candidate genes for aflatoxin production resistance in post-harvest seeds were identified. The study provided the first comprehensive report of the transcriptomes of post-harvest peanut seed in response to aflatoxin production, and enhanced the genomic resource database for 
peanut. Future functional analysis of the responsive genes will provide a better understanding of the molecular mechanism of defense against aflatoxin contamination in peanut and will facilitate identifying major candidate genes and molecular markers for improving resistance to aflatoxin contamination.

\section{Methods}

\section{Plant material and treatments}

Seeds of Zhonghua 6 and Zhonghua 12 were obtained from the Oil Crops Research Institute of Chinese Academy of Agricultural Sciences (CAAS-OCRI). Previous experiments showed that both Zhonghua 6 and Zhonghua 12 were susceptible to seed invasion by A. flavus in postharvest seeds; however, their resistance to aflatoxin production was highly different, with Zhonghua 6 being resistant and Zhonghua 12 susceptible [41]. The toxigenic $A$. flavus strain (AF2202) isolated from peanut was maintained in $20 \%$ glycerol $\left(-80{ }^{\circ} \mathrm{C}\right)$ at CAAS-OCRI. To prepare the A. flavus inoculation, conidia of AF2202 were taken from the stored sample and cultured on fresh potato dextrose agar medium at $29 \pm 1{ }^{\circ} \mathrm{C}$ for 7 days. Conidia were then collected and suspended in sterile water containing $0.05 \%$ Tween-80. The concentration of conidia in the suspension was determined using a haemocytometer.

Healthy post-harvest mature seeds of the R and S genotypes were selected for the experiments. All seeds were surface-sterilized by immersion in $70 \%$ ethanol for $1.0 \mathrm{~min}$, and rinsed with sterile distilled water three times for $5.0 \mathrm{~min}$ each. In the artificial inoculation treatments, $0.5 \mathrm{ml}$ spores suspension $\left(4.0 \times 10^{6} \mathrm{CFU} / \mathrm{ml}\right)$ was directly added to $10.0 \mathrm{~g}$ of peanut seeds in a sterile Petri plate. In the control, $0.5 \mathrm{ml} 0.05 \%$ Tween- 80 solution was added to the peanut seeds. Then, the inoculated samples and the control were placed in an incubator and cultured at $29 \pm 1{ }^{\circ} \mathrm{C}$ in darkness. Depending on the specific purpose of the experiment, the seeds were taken out to test aflatoxin content (five replications) or to extract RNA (two replications) after incubation for 1 to 10 days.

The incubated peanut seeds autoclaved at $121{ }^{\circ} \mathrm{C}$ for $30 \mathrm{~min}$, then dried at $110{ }^{\circ} \mathrm{C}$ for $60 \mathrm{~min}$. After cooling, the seeds of each experimental unit (10 g) were finely ground into powders, then were extracted using $50.0 \mathrm{ml}$ methanol-water $(55: 45 ; \mathrm{v} / \mathrm{v})$ in a flask. After shaking extraction (200 rpm, $30 \mathrm{~min})$ and filtration with quantitative filter paper, the filtrate $(10.0 \mathrm{ml})$ was collected in a $125 \mathrm{ml}$ flask, then diluted with $90 \mathrm{ml}$ methanol-water (55:45), blended and filtered with organic membrane $(0.45 \mu \mathrm{m})$. The purified extract $(1.0 \mathrm{ml})$ was collected in the glass tube and $10.0 \mu \mathrm{l}$ was prepared for high performance liquid chromatography (HPLC) analysis [5, 82]. HPLC analysis was performed with an Agilent 1200 HPLC system (USA) equipped with a fluorescence detector (G1321A) at wavelengths of $360 \mathrm{~nm}$ and $440 \mathrm{~nm}$ for excitation and emission, respectively. Chromatographic separation was performed on a $\mathrm{C}_{18}$ column $(150 \mathrm{~mm} \times 4.6 \mathrm{~mm}, 5 \mu \mathrm{l}$ particle size), with a methanol-water (45:55) mobile phase, at a flow rate of $0.7 \mathrm{ml} / \mathrm{min}$.

\section{RNA isolation and CDNA library construction}

Seeds of the $\mathrm{R}$ and $\mathrm{S}$ genotypes inoculated with A. flavus (treatment) and without inoculation (control) cultured for 1, 3 and 7 days were sampled for RNA isolation and cDNA library construction. Two replicates were prepared for each sample, resulting in 24 libraries that were used for transcriptome sequencing using the Illumina HiSeq2000 system at Novogene Bioinformatics Technology Co. Ltd., (Beijing, China).

Total RNA of post-harvest peanut seeds was isolated using the RNeasy ${ }^{\circ}$ Plant Mini Kit (QIAGEN), according to the manufacturer's protocol. All RNA samples were treated with RNase-free DNase I. The concentration and integrity of the pooled total RNA was checked using a NanoDrop ${ }^{\circ}$ 2000 spectrophotometer, a Qubit ${ }^{\circ}$ Fluorometer 2.0, and an Agilent 2100 bioanalyzer, to confirm that all samples had an RNA integrity number greater than 6.5. RNA quality detection, cDNA library construction, and Illumina deep sequencing were performed following the previous method [15, 18-20].

\section{Data filtering and de novo sequence assembly}

Raw data (raw reads) in the fastq format were first processed using in-house perl scripts. The raw data were then filtered by data-processing steps to generate original clean data via a process that included the removal of adapters, reads containing poly- $\mathrm{N}$ and low quality reads. For the samples infected with $A$. flavus, the original clean data contained a certain amount of $A$. flavus's transcriptomic data. All paired-end clean reads were aligned to the reference genome of A. flavus using Tophat (v2.0.7) with "mismatch 2" as the parameter [83], then the transcriptomic data of A. flavus were filtered out and to obtaind clean data of A. hypogaea. The calculation of Q20, Q30, GC content and the sequence duplication level, and all downstream analyses were used the clean data with high quality. After the clean data were generated, the assembled $A$. hypogaea reference genome was processed using Trinity software with min_kmer_cov set to 1 and other parameters set to default values [84].

\section{Functional annotation of unigenes}

For functional annotation, all assembled unigenes were annotated based on the following seven databases: $\mathrm{Nr}, \mathrm{Nt}$, KOG, Swiss-Prot, Pfam, GO, and KO. The unigenes were 
annotated in Nr, Nt and Swiss-Prot databases using NCBI blast 2.2.28+, with an E-value of $1.0 \times 10^{-5}$, and annotated in KOG database using NCBI blast 2.2.28+ with an Evalue of $1.0 \times 10^{-3}$. KAAS (rl40224) was applied to annotate the unigenes in the $\mathrm{KO}$ database with a cutoff $\mathrm{E}$-value of $1.0 \times 10^{-10}$ [85]. The unigenes annotated in the Pfam database using HMMScan (HMMER 3) with an E-value of 0.01 [86]. In addition, the unigenes were assigned GO annotations using Blast2go (b2g4pipe_v2.5) with an E-value of $1.0 \times 10^{-6}$ [87].

\section{Expression analysis and enrichment analysis}

Gene expression levels were estimated using the RNA-Seq with Expectation-Maximization (RSEM) (rsem-1.2.0) method for each library [24]. The index of the assembled reference genome was built using Bowtie (mismatch 2), and clean reads of each library were aligned to the assembled reference genome using RSEM. RSEM then counted the read numbers mapped to each gene. Then, the FPKM of each gene was calculated based on the length of the gene and read count mapped to it [83].

Differential expression analysis of two samples was performed using the DESeq R package (1.12.0). DESeq provide statistics to determine differential expression in gene expression data using a model based on the negative binomial distribution. The resulting $p$ values were adjusted using the Benjamini and Hochberg's approach to control the false discovery rate. In this study, unigenes with an adjusted $p(q)$ value $<0.05$ found by DESeq were considered as differentially expressed [25].

GO enrichment analysis of the DEGs was conducted using GOseq R packages based on Wallenius non-central hyper-geometric distribution [26], which can adjust for gene length bias in DEGs. GO terms with corrected $p(q)$ value $<0.05$ were considered significantly enriched among the DEGs. KEGG pathway database records the network of molecular interactions in the cells and variants specific to particular organisms, with molecular information mainly from large-scale molecular datasets generated by genome sequencing and other high-throughput experimental technologies [30]. KOBAS (v2.0.12) software was used to enrich the DEGs in the KEGG pathways [88]. A corrected $p(q)$ value $<0.05$ was the threshold for significantly enriched KEGG pathways in this study.

\section{qRT-PCR analysis}

To validate the repeatability and reproducibility of gene expression data obtained by RNA-seq in A. hypogaea, we randomly selected 20 unigenes for validation by qRT-PCR, as described previously $[15,18-20]$. Independent RNA of the control peanut seeds without $A$. flavus inoculation of $\mathrm{R}$ and $\mathrm{S}$ genotypes, which were incubated for 1,3 , and 7 days at $29 \pm 1{ }^{\circ} \mathrm{C}$, was prepared for qRT-PCR analysis. RNA extraction and quality control were performed as described above. Genespecific primer pairs (Additional file 8) were designed according to the sequences of the 20 genes, using the GenScript Real-time PCR Primer Design program available online (https://www.genscript.com/ssl-bin/app/primer). To ensure accuracy, each primer was run with three replications on the same plate, with a negative control that lacked template cDNA to detect nonspecific products. Candidate genes were tested in triplicate wells and in three replicate experiments. The relative expression levels of the genes were calculated using the $2^{-\Delta \Delta C t}$ method $[89,90]$, which represents the $C_{T}$ (cycle threshold) difference between the reference Actin gene and the target gene product [15].

\section{Availability of supporting data}

The sequencing data generated in this study have been deposited in NCBI's Short Read Archive database (SRA, http://www.ncbi.nlm.nih.gov/Traces/sra_sub/sub.cgi) and are accessible through SRA series accession number SRP061959 (BioProject ID: PRJNA291797).

\section{Additional files}

Additional file 1: Classification of raw reads. Raw reads including clean reads (purple), adapter sequences (green), reads containing undefined nucleotides (N's) (yellow), and low quality reads (orange) generated from Illumina RNA-sequencing (RNA-seq). (RAR $1485 \mathrm{~kb}$ )

Additional file 2: The length distribution of unigenes and transcripts. (A) Transcript length distribution; (B) Unigene length distribution. (RAR $97 \mathrm{~kb}$ )

Additional file 3: Summary of GO, KOG, and KEGG classifications of the assembled unigenes. (XLSX $32 \mathrm{~kb}$ )

Additional file 4: Summary of the uniquely mapped reads in each library. (XLSX $10 \mathrm{~kb}$ )

Additional file 5: Distribution of unigenes expression in each library. NEGs: the number of expressed unigenes in each library. NSEGs: the number of specifically expressed unigenes in each library. (DOCX $17 \mathrm{~kb}$ )

Additional file 6: Statistics analysis of FPKM value of all unigenes. (XLSX $16479 \mathrm{~kb}$ )

Additional file 7: Pearson correlation analysis between samples. (RAR $473 \mathrm{~kb}$ )

Additional file 8: Primer information for genes used to validate the FPKM-based expression data using qRT-PCR. (XLSX $10 \mathrm{~kb}$ )

Additional file 9: Quantitative real-time PCR validations of the FPKM-based expression of unigenes by RNA-seq. Note: RE indicates relative expression levels of unigenes by qRT-PCR. (RAR $18 \mathrm{~kb}$ )

Additional file 10: Comparison analysis of differentially expressed genes. (DOCX $16 \mathrm{~kb}$ )

Additional file 11: Differentially expressed unigenes analysis and annotation in each comparison. (XLSX $16087 \mathrm{~kb}$ )

Additional file 12: Number of DEGs in the control and A. flavus inoculated samples of peanut seeds. The numbers of DEGs in one sample or comparison are shown in each circle. The numbers of DEGs between the two samples or comparisons are shown in the overlapping regions. (RAR $118 \mathrm{~kb}$ )

Additional file 13: Hierarchical clustering of the differentially expressed unigenes in the peanut seeds. The blue, white, and red 
bands indicate low, intermediate, and high gene expression quantity, respectively. (RAR $2787 \mathrm{~kb}$ )

Additional file 14: Hierarchical clustering of the DEGs in two biological replicates of the peanut seeds. A, B and C showed the results of hierarchical clustering analysis at three culture time points, respectively. The blue, white, and red bands indicate low, intermediate, and high gene expression quantity, respectively. (RAR $110 \mathrm{~kb}$ )

Additional file 15: GO functional enrichment analysis of differentially expressed genes when peanut seeds respond to aflatoxin production by $A$. flavus. (XLSX $78 \mathrm{~kb}$ )

Additional file 16: KEGG pathway enrichment analysis of differentially expressed genes when peanut seeds respond to aflatoxin production by $A$. flavus. (XLSX $12 \mathrm{~kb}$ )

Additional file 17: Expression analysis of peanut defense-related genes in response to aflatoxin production by A. flavus. (XLSX $373 \mathrm{~kb}$ )

\section{Competing interests}

The authors declare that they have no competing interests.

\section{Authors' contributions}

HMW conceived, designed and performed the experiments, analyzed and interpreted the results, and wrote the manuscript. BSL, YL and LYW conceived and designed the experiments. LYY assisted for culturing and inoculation A. flavus. JWL and XPR assisted for aflatoxin content testing. HFJ, WG and XFD corrected the written English of Background, Results and Disscusion, respectively. All authors have read and approved the final manuscript.

\section{Acknowledgments}

This research was funded by the National Basic Research Program of China (2013CB127803 and 2011CB109304), National High Technology Research and Development Program of China (No. 2013AA102602), the National Natural Science Foundation of China (No. 31371662 and 31461143022), and the China Agriculture Research System (CARS-14).

\section{Author details}

${ }^{1}$ Key Laboratory of Oil Crop Biology of the Ministry of Agriculture, Oil Crops Research Institute of Chinese Academy of Agricultural Sciences, Wuhan 430062, China. ${ }^{2}$ Chinese Academy of Agricultural Sciences-International Crop Research Institute for the Semi-Arid Tropics Joint Laboratory for Groundnut Aflatoxin Management, Oil Crops Research Institute of Chinese Academy of Agricultural Sciences, Wuhan 430062, China. ${ }^{3}$ Institute of Agro-Products Processing Science and Technology, Chinese Academy of Agricultural Sciences, Beijing 100193, China.

Received: 8 September 2015 Accepted: 17 February 2016

Published online: 27 February 2016

\section{References}

1. Torres AM, Barros GG, Palacios SA, Chulze SN, Battilani P. Review on pre- and post-harvest management of peanuts to minimize aflatoxin contamination. Food Res Int. 2014;62:11-9.

2. Bhatnagar-Mathur P, Sunkara S, Bhatnagar-Panwar M, Waliyar F, Sharma KK. Biotechnological advances for combating Aspergillus flavus and aflatoxin contamination in crops. Plant Sci. 2015;234:119-32.

3. Liang X, Luo M, Guo B. Resistance mechanisms to Aspergillus flavus infection and aflatoxin contamination in peanut (Arachis hypogaea). Plant Pathol J. 2006;5(1):115-24.

4. Guo B, Chen Z-Y, Lee RD, Scully BT. Drought stress and preharvest aflatoxin contamination in agricultural commodity: genetics, genomics and proteomics. J Integr Plant Biol. 2008;50(10):1281-91.

5. Ding X, Li P, Bai Y, Zhou H. Aflatoxin B 1 in post-harvest peanuts and dietary risk in China. Food Control. 2012;23(1):143-8.

6. Passone MA, Ruffino M, Ponzio V, Resnik S, Etcheverry MG. Postharvest control of peanut Aspergillus section Flavi populations by a formulation of food-grade antioxidants. Int J Food Microbiol. 2009;131(2-3):211-7.

7. Probst C, Schulthess F, Cotty PJ. Impact of Aspergillus section Flavi community structure on the development of lethal levels of aflatoxins in Kenyan maize (Zea mays). J Appl Microbiol. 2010;108(2):600-10.
8. Williams JH, Phillips TD, Jolly PE, Stiles JK, Jolly CM, Aggarwal D. Human aflatoxicosis in developing countries: a review of toxicology, exposure, potential health consequences, and interventions. Am J Clin Nutr. 2004;80(5):1106-22.

9. Gong Y, Hounsa A, Egal S, Turner PC, Sutcliffe AE, Hall AJ, Cardwell K, Wild CP. Postweaning exposure to aflatoxin results in impaired child growth: a longitudinal study in Benin, West Africa. Environ Health Perspect. 2004;112(13):1334-8

10. Turner PC, Moore SE, Hall AJ, Prentice AM, Wild CP. Modification of immune function through exposure to dietary aflatoxin in Gambian children. Environ Health Perspect. 2003;111(2):217-20.

11. Cary JW, Rajasekaran K, Brown RL, Luo M, Chen Z-Y, Bhatnagar D. Developing resistance to aflatoxin in maize and cottonseed. Toxins. 2011;3(6):678-96.

12. Nigam SN, Waliyar F, Aruna R, Reddy SV, Kumar PL, Craufurd PQ, Diallo AT, Ntare BR, Upadhyaya HD. Breeding peanut for resistance to aflatoxin contamination at ICRISAT. Peanut Sci. 2009;36(1):42-9.

13. Wang Z, Yan S, Liu C, Chen F, Wang T. Proteomic analysis reveals an aflatoxin-triggered immune response in cotyledons of Arachis hypogaea infected with Aspergillus flavus. J Proteome Res. 2012;11(5):2739-53.

14. Zhang HL, Scharfenstein L, Zhang DH, Chang PK, Montalbano BG, Guo BZ, Meng XJ, Yu JJ. Peanut resistance gene expression in response to Aspergillus flavus infection during seed germination. J Phytopathol. 2015;163(3):212-21.

15. Chen Y, Ren X, Zhou X, Huang L, Yan L, Lei Y, Liao B, Huang J, Huang S, Wei W. Dynamics in the resistant and susceptible peanut (Arachis hypogaea L.) root transcriptome on infection with the Ralstonia solanacearum. BMC Genomics. 2014;15:1078.

16. Zhu W, Chen X, Li H, Zhu F, Hong Y, Varshney RK, Liang X. Comparative transcriptome analysis of aerial and subterranean pods development provides insights into seed abortion in peanut. Plant Mol Biol. 2014;85(4-5):395-409.

17. Geng L, Duan X, Liang C, Shu C, Song F, Zhang J. Mining tissue-specific contigs from peanut (Arachis hypogaea L.) for promoter cloning by deep transcriptome sequencing. Plant Cell Physiol. 2014;55(10):1793-801.

18. Li X, Lu J, Liu S, Liu X, Lin Y, Li L. Identification of rapidly induced genes in the response of peanut (Arachis hypogaea) to water deficit and abscisic acid. BMC Biotechnol. 2014;14:58

19. Yin D, Wang Y, Zhang X, Li H, Lu X, Zhang J, Zhang W, Chen S. De novo assembly of the peanut (Arachis hypogaea L.) seed transcriptome revealed candidate unigenes for oil accumulation pathways. Plos One. 2013;8(9):e73767.

20. Guimaraes PM, Brasileiro ACM, Morgante CV, Martins ACQM, Pappas G, Silva Junior OB, Togawa R, Leal-Bertioli SCM, Araujo ACG, Moretzsohn MCM. Global transcriptome analysis of two wild relatives of peanut under drought and fungi infection. BMC Genomics. 2012;13:387.

21. McGettigan PA. Transcriptomics in the RNA-seq era. Curr Opin Chem Biol. 2013;17(1):4-11.

22. Holbrook CC, Stalker HT. Peanut breeding and genetic resources. Plant Breed Rev. 2003;22:297-356.

23. Trapnell C, Williams BA, Pertea G, Mortazavi A, Kwan G, van Baren MJ, Salzberg SL, Wold BJ, Pachter L. Transcript assembly and quantification by RNA-Seq reveals unannotated transcripts and isoform switching during cell differentiation. Nat Biotechnol. 2010;28(5):511-5.

24. Li B, Dewey CN. RSEM: accurate transcript quantification from RNA-Seq data with or without a reference genome. BMC Bioinformatics. 2011;12(1):323.

25. Anders S, Huber W. Differential expression analysis for sequence count data. Genome Biol. 2010;11(10):R106.

26. Young MD, Wakefield MJ, Smyth GK, Oshlack A. Gene ontology analysis for RNA-seq: accounting for selection bias. Genome Biol. 2010;11:2.

27. Naoumkina MA, Zhao Q, Gallego-Giraldo L, Dai X, Zhao PX, Dixon RA. Genome-wide analysis of phenylpropanoid defence pathways. Mol Plant Pathol. 2010;11(6):829-46.

28. Swarbrick PJ, Schulze-Lefert P, Scholes JD. Metabolic consequences of susceptibility and resistance (race-specific and broad-spectrum) in barley leaves challenged with powdery mildew. Plant Cell Environ. 2006;29(6):1061-76.

29. Heil M, BOSTOCK RM. Induced systemic resistance (ISR) against pathogens in the context of induced plant defences. Ann Bot-London. 2002;89(5):503-12.

30. Kanehisa M, Araki M, Goto S, Hattori M, Hirakawa M, Itoh M, Katayama T, Kawashima S, Okuda S, Tokimatsu T. KEGG for linking genomes to life and the environment. Nucleic Acids Res. 2008;36 suppl 1:D480-4. 
31. Xie C, Mao X, Huang J, Ding Y, Wu J, Dong S, Kong L, Gao G, Li CY, Wei L. KOBAS 2.0: a web server for annotation and identification of enriched pathways and diseases. Nucleic Acids Res. 2011;39(suppl):W316-22.

32. Asters $M C$, Williams WP, Perkins AD, Mylroie JE, Windham GL, Shan X. Relating significance and relations of differentially expressed genes in response to Aspergillus flavus infection in maize. Sci Rep-UK. 2014;4:4815.

33. Wang $T$, Chen XP, Li HF, Liu HY, Hong YB, Yang QL, Chi XY, Yang Z, Yu SL, $\mathrm{Li}$ L. Transcriptome identification of the resistance-associated genes (RAGs) to Aspergillus flavus infection in pre-harvested peanut (Arachis hypogaea). Funct Plant Biol. 2013;40(3):292-303

34. Dolezal AL, Obrian GR, Nielsen DM, Woloshuk CP, Boston RS, Payne GA. Localization, morphology and transcriptional profile of Aspergillus flavus during seed colonization. Mol Plant Pathol. 2013;14(9):898-909.

35. Guo B, Chen X, Dang P, Scully BT, Liang X, Holbrook CC, Yu J, Culbreath AK Peanut gene expression profiling in developing seeds at different reproduction stages during Aspergillus parasiticus infection. BMC Dev Biol. 2008:8:12.

36. Vogt T. Phenylpropanoid biosynthesis. Mol Plant. 2010;3(1):2-20.

37. Chen X, Zhu W, Azam S, Li H, Zhu F, Li H, Zhang E, Wu H. Deep sequencing analysis of the transcriptomes of peanut aerial and subterranean young pods identifies candidate genes related to early embryo abortion. Plant Biotechnol J. 2013;11(1):115-27.

38. Zhang J, Liang S, Duan J, Wang J, Chen S, Cheng Z, Zhang Q, Liang X, Li Y. De novo assembly and vharacterisation of the transcriptome during seed development, and generation of genic-SSR markers in peanut (Arachis hypogaea L.). BMC Genomics. 2012;13:90.

39. Long Y, Zhang J, Tian X, Wu S, Zhang Q, Zhang J, Dang Z, Pei X. De novo assembly of the desert tree Haloxylon ammodendron (C. A. Mey.) based on RNA-Seq data provides insight into drought response, gene discovery and marker identification. BMC Genomics. 2014;15:1111.

40. Coulombe RA, Hawkins LK, Mylroie JE, Oliveira DA, Smith JS, Ozkan S, Windham GL, Williams WP, Warburton ML. Characterization of the maize chitinase genes and their effect on Aspergillus flavus and aflatoxin accumulation resistance. Plos One. 2015;10(6):e0126185.

41. Wang H, Huang J, Lei Y, Yan L, Wang S, Jiang H, Ren X, Lou Q, Liao B. Relationship of resveratrol content and resistance to aflatoxin accumulation caused by Aspergillus flavus in peanut seeds. Acta Agron Sin. 2012;38(10):1875-83.

42. Tsitsigiannis DI, Kunze S, Willis DK, Feussner L, Keller NP. Aspergillus infection inhibits the expression of peanut 13S-HPODE-forming seed lipoxygenases. Mol Plant Microbe Interact. 2005;18(10):1081-9.

43. Yan Z, Thomas L, Mingliang X. The genetic and molecular basis of plant resistance to pathogens. J Genet Genomics. 2013;40(1):23-35.

44. Amaike S, Keller NP. Aspergillus flavus. In: VanAlfen NK, Bruening G, Leach JE, editors. Annu Rev Phytopathol, vol. 49. 2011. p. 107-33.

45. Kalunke RM, Tundo S, Benedetti M, Cervone F, De Lorenzo G, D'Ovidio R. An update on polygalacturonase-inhibiting protein (PGIP), a leucine-rich repeat protein that protects crop plants against pathogens. Front Plant Sci. 2015;6:146.

46. Federici L, Di Matte A, Fernandez-Recio J, Tsernoglou D, Cervone F. Polygalacturonase inhibiting proteins: players in plant innate immunity? Trends Plant Sci. 2006;11(2):65-70.

47. Ferrari S, Savatin DV, Sicilia F, Gramegna G, Cervone F, De Lorenzo G. Oligogalacturonides: plant damage-associated molecular patterns and regulators of growth and development. Front Plant Sci. 2013;4:49.

48. Mitra SK, Chen R, Dhandaydham M, Wang X, Blackburn RK, Kota U, Goshe MB, Schwartz D, Huber SC, Clouse SD. An autophosphorylation site database for leucine-rich repeat receptor-like kinases in Arabidopsis thaliana. Plant J. 2015:82(6):1042-60

49. Park S, Moon J-C, Park YC, Kim J-H, Kim DS, Jang CS. Molecular dissection of the response of a rice leucine-rich repeat receptor-like kinase (LRR-RLK) gene to abiotic stresses. J Plant Physiol. 2014;171(17):1645-53.

50. Xu J, Zhang S. Mitogen-activated protein kinase cascades in signaling plant growth and development. Trends Plant Sci. 2015;20(1):56-64.

51. Meng $X$, Zhang S. MAPK cascades in plant disease resistance signaling. Annu Rev Phytopathol. 2013;51:245-66.

52. Pandey SP, Somssich IE. The role of WRKY transcription factors in plant immunity. Plant Physiol. 2009;150(4):1648-55.

53. Seo $E$, Choi D. Functional studies of transcription factors involved in plant defenses in the genomics era. Brief Funct Genomics. 2015;14(4):260-7.

54. Ulker B, Somssich IE. WRKY transcription factors: from DNA binding towards biological function. Curr Opin Plant Biol. 2004;7(5):491-8.
55. ZG E, Zhang YP, Zhou JH, Wang L. Roles of the bZIP gene family in rice. Genet Mol Res. 2014;13(2):3025-36.

56. Hong S-Y, Roze LV, Linz JE. Oxidative stress-related transcription factors in the regulation of secondary metabolism. Toxins. 2013;5(4):683-702.

57. Licausi F, Ohme-Takagi M, Perata P. APETALA/Ethylene Responsive Factor (AP2/ERF) transcription factors: mediators of stress responses and developmental programs. New Phytol. 2013;199(3):639-49.

58. Kraus WL. PARPs and ADP-Ribosylation: 50 Years ... Counting. Mol Cell. 2015;58(6):902-10

59. Pelletan LE, Suhaiman L, Vaquer CC, Bustos MA, De Blas GA, Vitale N, Mayorga LS, Belmonte SA. ADP Ribosylation factor 6 (ARF6) promotes acrosomal exocytosis by modulating lipid turnover and rab3A activation. J Biol Chem. 2015;290(15):9823-41.

60. Bari $\mathrm{R}$, Jones J. Role of plant hormones in plant defence responses. Plant Mol Biol. 2009:69(4):473-88.

61. Xin M, Wang X, Peng H, Yao Y, Xie C, Han Y, Ni Z, Sun Q. T Transcriptome comparison of susceptible and resistant wheat in response to powdery mildew infection. Genomics Proteomics Bioinformatics. 2012;10(2):94-106.

62. Fan J, Hill L, Crooks C, Doerner P, Lamb C. Abscisic acid has a key role in modulating diverse plant-pathogen interactions. Plant Physiol. 2009;150(4):1750-61.

63. Bleecker $\mathrm{AB}$, Kende $\mathrm{H}$. Ethylene: a gaseous signal molecule in plants. Annu Rev Cell Dev Biol. 2000;16:1-18.

64. Huang JQ, Jiang HF, Zhou YQ, Lei Y, Wang SY, Liao BS. Ethylene inhibited aflatoxin biosynthesis is due to oxidative stress alleviation and related to glutathione redox state changes in Aspergillus flavus. Int J Food Microbiol. 2009;130(1):17-21

65. Seyfferth C, Tsuda K. Salicylic acid signal transduction: the initiation of biosynthesis, perception and transcriptional reprogramming. Front Plant Sci. 2014;5:697.

66. Panahirad S, Zaare-Nahandi F, Mohammadi N, Alizadeh-Salteh S, Safaie N. Effects of salicylic acid on Aspergillus flavus infection and aflatoxin B-1 accumulation in pistachio (Pistacia vera L.) fruit. J Sci Food Agric. 2014;94(9):1758-63.

67. Gu LJ, Si WN, Zhao LN, Yang SH, Zhang XH. Dynamic evolution of NBS-LRR genes in bread wheat and its progenitors. Mol Genet Genomics. 2015;290(2):727-38.

68. Cletus J, Balasubramanian V, Vashisht D, Sakthivel N. Transgenic expression of plant chitinases to enhance disease resistance. Biotechnol Lett. 2013;35(11):1719-32

69. Singh HR, Deka M, Das S. Enhanced resistance to blister blight in transgenic tea (Camellia sinensis) by overexpression of class I chitinase gene from potato (Solanum tuberosum). Funct Integr Genomics. 2015;15(4):461-80.

70. Dang L, Van Damme EJM. Toxic proteins in plants. Phytochem. 2015;117:51-64.

71. Tintu I, Abhilash J, Dileep KV, Augustine A, Haridas M, Sadasivan C. A Lectin from spatholobus parviflorus inhibits Aspergillus flavus-amylase: enzyme kinetics and thermodynamic studies. Chem Biol Drug Des. 2014;84(1):116-22

72. Gill SS, Tuteja N. Reactive oxygen species and antioxidant machinery in abiotic stress tolerance in crop plants. Plant Physiol Biochem. 2010;48(12):909-30.

73. Yan S, Liang Y, Zhang J, Chen Z, Liu C-M. Autoxidated linolenic acid inhibits aflatoxin biosynthesis in Aspergillus flavus via oxylipin species. Fungal Genet Biol. 2015;81:229-37.

74. Sels J, Mathys J, De Coninck BMA, Cammue BPA, De Bolle MFC. Plant pathogenesis-related (PR) proteins: a focus on PR peptides. Plant Physiol Biochem. 2008:46(11):941-50.

75. Agarwal P, Agarwal PK. Pathogenesis related-10 proteins are small, structurally similar but with diverse role in stress signaling. Mol Biol Rep. 2014:41(2):599-611.

76. Chen ZY, Brown RL, Damann KE, Cleveland TE. PR10 expression in maize and its effect on host resistance against Aspergillus flavus infection and aflatoxin production. Mol Plant Pathol. 2010;11(1):69-81.

77. Chen Z-Y, Brown R, Rajasekaran K, Damann K, Cleveland T. Identification of a maize kernel pathogenesis-related protein and evidence for its involvement in resistance to Aspergillus flavus infection and aflatoxin production. Phytopathol. 2006;96(1):87-95.

78. Mitsuhara I, Iwai T, Seo S, Yanagawa Y, Kawahigasi H, Hirose S, Ohkawa Y, Ohashi Y. Characteristic expression of twelve rice PR1 family genes in 
response to pathogen infection, wounding, and defense-related signal compounds (121/180). Mol Genet Genomics. 2008;279(4):415-27.

79. Liu J, Osbourn A, Ma P. MYB transcription factors as regulators of phenylpropanoid metabolism in plants. Mol Plant. 2015;8(5):689-708.

80. Norton RA. Inhibition of aflatoxin B-1 biosynthesis in Aspergillus flavus by anthocyanidins and related flavonoids. J Agric Food Chem. 1999:47(3):1230-5.

81. DeLucca II AJ, Palmgren MS, Daigle DJ. Depression of aflatoxin production by flavonoid-type compounds from peanut shells. Phytopathol. 1987;77(11):1560-3.

82. Wang HM, Lei Y, Yan LY, Cheng K, Dai XF, Wan LY, Guo W, et al. Deep sequencing analysis of transcriptomes in Aspergillus flavus in response to resveratrol. BMC Microbiol. 2015;15.

83. Trapnell C, Pachter L, Salzberg SL. TopHat: discovering splice junctions with RNA-Seq. Bioinformatics. 2009;25(9):1105-11.

84. Grabherr MG, Haas BJ, Yassour M, Levin JZ, Thompson DA, Amit I, Adiconis X, Fan L, Raychowdhury R, Zeng Q, et al. Full-length transcriptome assembly from RNA-Seq data without a reference genome. Nat Biotechnol. 2011;29(7):644-52.

85. Moriya Y, Itoh M, Okuda S, Yoshizawa AC, Kanehisa M. KAAS: an automatic genome annotation and pathway reconstruction server. Nucleic Acids Res. 2007;35 suppl 2:W182-5.

86. Punta M, Coggill PC, Eberhardt RY, Mistry J, Tate J, Boursnell C, Pang N, Forslund K, Ceric G, Clements J. The Pfam protein families database. Nucleic Acids Res. 2012;40(D1):D290-301.

87. Götz S, García-Gómez JM, Terol J, Williams TD, Nagaraj SH, Nueda MJ, Robles M, Talón M, Dopazo J, Conesa A. High-throughput functional annotation and data mining with the Blast2GO suite. Nucleic Acids Res. 2008:36(10):3420-35.

88. Mao XZ, Cai T, Olyarchuk JG, Wei LP. Automated genome annotation and pathway identification using the KEGG Orthology (KO) as a controlled vocabulary. Bioinformatics. 2005;21(19):3787-93.

89. Livak KJ, Schmittgen TD. Analysis of relative gene expression data using real-time quantitative PCR and the $2(T)(-$ Delta Delta C) method. Methods. 2001;25(4):402-8.

90. Pfaffl MW. A new mathematical model for relative quantification in real-time RT-PCR. Nucleic Acids Res. 2001;29(9), e45.

\section{Submit your next manuscript to BioMed Central and we will help you at every step:}

- We accept pre-submission inquiries

- Our selector tool helps you to find the most relevant journal

- We provide round the clock customer support

- Convenient online submission

- Thorough peer review

- Inclusion in PubMed and all major indexing services

- Maximum visibility for your research

Submit your manuscript at www.biomedcentral.com/submit

) Biomed Central 